\title{
Anaesthetic management of a patient with Jervell and Langee Nielson Syndrome (a long QT variant) for cochlear implantation
}

\author{
Prisley Fernando ${ }^{1 *}$, P.A.N. Lochanie ${ }^{2}$ \\ Registrar in Anaesthesiology ${ }^{1^{*}}$, National Hospital of Sri Lanka, Colombo \\ Consultant Anaesthetist ${ }^{2}$, Lady Ridgeway Hospital, Colombo, Sri Lanka
}

\begin{abstract}
Development of life-threatening arrhythmia is the main concern in the perioperative period of a patient with LQTS, which significantly increases morbidity and mortality. Specific pre, intra and postoperative measures will reduce the risk of life-threatening arrhythmia up to a considerable level. We report a successful perioperative management of a cochlear implant in a $2 \frac{1}{2}$ year old child with LQTS.
\end{abstract}

Keywords: Long QT syndrome (LQTS); Torsade de pointes (Tdp); corrected QT interval $\left(\mathrm{QT}_{\mathrm{c}}\right)$

\section{Introduction}

Children with LQTS coming for non-cardiac surgery pose a significant challenge to the anaesthetist. They are at high risk of Torsade de pointes (Tdp), polymorphic ventricular tachycardia and sudden cardiac death during the perioperative period. Although most patients have an uneventful perioperative course, it is imperative that they are properly optimised and all potential precipitance are avoided to minimise serious complications

\section{Case report}

A $2 \frac{1}{2}$ year old boy with sensorineural deafness presented for a cochlear implant. He was incidentally diagnosed to have LQTS at the age of 3 months when an ECG done before starting propranolol for a small haemangioma in the neck. He never had syncopal attacks or seizure episodes. He did not have any syndromic features and structural heart diseases were excluded by $2 \mathrm{D}$ echocardiogram. All acquired causes of LQTS was excluded. He had never undergone anaesthesia before and did not have a family history of LQTS or sudden cardiac death in first degree relatives.
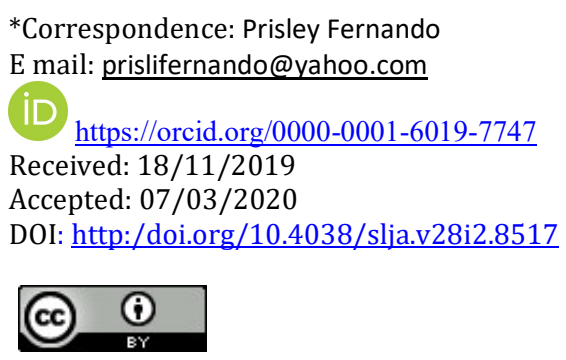

In pre-op 12 lead $\mathrm{ECG}, \mathrm{QT}_{\mathrm{c}}$ was $460 \mathrm{~ms}$ and there were no ST segment or $\mathrm{T}$ wave abnormalities. Serum electrolytes were within normal limits.

Risk assessment was done using Schwartz score. This patient had an intermediate probability of LQTS with a score of 2.5.

He was referred to the cardiac electrophysiology team 2 months prior to surgery and bisoprolol $0.625 \mathrm{mg}$ was started as prophylaxis against inadvertent perioperative cardiac events.

Strict fasting guidelines were adhered in order to avoid dehydration and irritability. Pre-emptively oral paracetamol $20 \mathrm{mg} / \mathrm{kg}$ was given 2 hours prior. Prophylactic antibiotic (IV cefotaxime 30 $\mathrm{mg} / \mathrm{kg}$ ) was given 1 hour prior to surgery. ICU bed was reserved for post operative care.

\section{Anaesthetic management}

Anaesthetic workstation, drugs and defibrillator were checked. Monitoring was established according to AAGBI standard and $\mathrm{QT}_{\mathrm{C}}$ from lead II of 3 lead ECG was monitored. Intravenous induction was preferred mode of induction using i.v. fentanyl $2 \mathrm{mcg} / \mathrm{kg}$, propofol $3 \mathrm{mg} / \mathrm{kg}$ and atracurium $0.5 \mathrm{mg} / \mathrm{kg}$. After intubation baby was ventilated in pressure controlled mode with $\mathrm{O}_{2}, \mathrm{~N}_{2} \mathrm{O}$ and isoflurane with a PEEP of $3 \mathrm{cmH}_{2} \mathrm{O}$, pressure limit of 18 $\mathrm{cmH}_{2} \mathrm{O}, \mathrm{FiO}_{2}$ of 0.5 , respiratory rate of $14 / \mathrm{min}$. Ventilatory targets were; tidal volume of 5-7 $\mathrm{ml} / \mathrm{kg}$, end tidal $\mathrm{CO}_{2}\left(\mathrm{ETCO}_{2}\right)$ was $35-40$ $\mathrm{mmHg}$ and $\mathrm{SpO}_{2}>97 \%$ and to avoid Valsalva effect. MAC was maintained at 0.8 to 1 . 
Target haemodynamic were; heart rate of 80 100 beats / $\mathrm{min}$, systolic blood pressure of 65 $80 \mathrm{mmHg}$ and to avoid arrhythmias. Ringer's lactate was given at $10 \mathrm{ml} / \mathrm{kg}$ in 1 st hour and 5 $\mathrm{ml} / \mathrm{kg}$ in subsequent hours.

Patient was warmed using forced air warming blanket and core temperature was monitored. Capillary blood sugar was $127 \mathrm{mg} / \mathrm{dl}$. Muscle relaxation was avoided after the intubation dose since facial nerve monitoring was planned.

Multimodal analgesia was offered with intravenous infusion of remifentanil 0.1$0.25 \mathrm{mcg} / \mathrm{kg} / \mathrm{min}$ intra operatively together with paracetamol and diclofenac sodium suppositories. Morphine $0.1 \mathrm{mg} / \mathrm{kg}$ was given i.v. 30 minutes prior to the end of the surgery and remifentanil was tailed off. Dexamethasone was given i.v. to reduce post-operative nausea and vomiting.

Surgery lasted for 2 hours and at the end of the surgery, spontaneous breathing was obtained and extubated after full recovery. Reversal agents were avoided. He was admitted to the ICU for monitoring and optimum pain relief.

Intraoperative period was uneventful and the $\mathrm{QT}_{\mathrm{C}}$ remained in the range of $445 \mathrm{~ms}-455 \mathrm{~ms}$ throughout the procedure.

\section{Discussion}

Patients with LQTS with or without treatment coming for anaesthesia carries a significant risk of refractory malignant arrhythmias. It is a disorder of cardiac ion channel leading to prolonged duration of action potential. The normal range of heart rate corrected QT interval varies with age and sex. 99th percentile value of QTc for $2 \frac{1}{2}$ years is $455 \mathrm{~ms}$.

At the time of diagnosis our patient's QTc was $457 \mathrm{~ms}$. Increasingly prolonged QTc values are at higher risk of potentially lethal arrhythmias. ${ }^{1}$ Diagnostic evaluation for LQTS can be done using Schwartz score. ${ }^{2}$

Score $\geq 3.5$ indicates high probability of LQTS.

Score 1.5 to 3.5 ; intermediate probability and a score $\leq 1.5$ - low probability. ${ }^{2}$

LQTS can be inherited or acquired. Prevalence of congenital LQTS is 1 in 2500 live births. ${ }^{1}$ About 17 of LQTS susceptible genes have been recognised. Most common LQTS1 include 2 genetic variants, Romano Ward syndrome
(RWS) and Jervell and Langee Nielson syndrome (JLNS). JLNS is homozygous autosomal recessive rarer form of LQTS, typically associated with deafness of sensorineural origin and a high incidence of cardiac events which is compatible with this patient. Responsible KCNQ1 gene codes the non functional $\alpha$ subunit of voltage gated potassium channel and produce slow rectifier $\mathrm{K}^{+}$current in both myocardium and cochlea. It is common in boys $<10$ years and associated with exercise induced cardiac events. ${ }^{3}$

QT prolongation can increase susceptibility of syncope, cardiogenic seizures, cardiac arrest and sudden cardiac death because of its characteristic arrhythmia - Tdp. ${ }^{1}$

Main target of anaesthesia for LQTS includes avoidance of precipitating factors and immediate recognition of Tdp.

\section{Membrane potential(mv)}

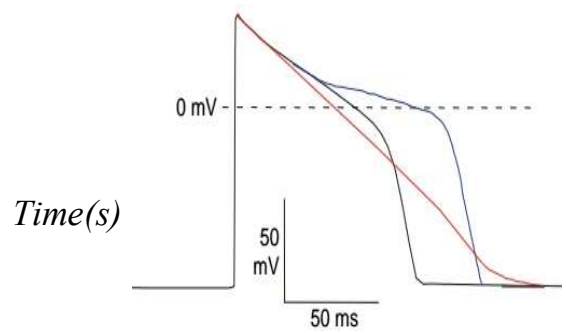

Cardiac action potential normal and with LQTS (black line - normal action potential, blue and red lines - abnormal action potentials)

Drugs and pathophysiological states created by anaesthesia which precipitate LQTS would prolong the duration of action potential either by a parallel shift or by triangulation of the final repolarization. The triangulation likely to cause arrhythmias. ${ }^{1,4}$

\section{Preoperative preparations}

Continuation of $\beta$ blocker therapy would not provide complete protection but reduces the risk of Tdp. Risk of bronchospasm with propranolol and unavailability of nadolol would have given the best option of bisoprolol in this patient.

Avoidance of "noncompliance" or "medication failure" by concurrent use of drugs that prolong QT time can provoke life threatening arrhythmias. $^{2}$ Almost always medication lists should be assessed. Treatment options would be implantation of cardioverter defibrillator (ICD) 
or left cardiac sympathetic denervation (LCSD) if the patients cannot tolerate beta blockers. LCSD includes left high thoracic sympathectomy and ablation of lower half of the stellate ganglion at T2-T4 which reduces the release of norepinephrine and increases the threshold level for ventricular fibrillation. ${ }^{1}$ If ICD is in place it should be checked before surgery.

Oral midazolam can be given as an anxiolytic to reduce associated sympathetic activation. But chloral hydrate and dexmedetomidine should be avoided because of the prolongation of QT. ${ }^{1}$

\section{Intraoperative management}

Prevention of excessive sympathetic stimulation and avoidance of precipitating factors of QT prolongation is the aim of perioperative period. Cefotaxime was selected as prophylactic antibiotics. Macrolides and fluoroquinolones can affect on QT time.

All possible efforts were taken to alleviate the anxiety during the time of entrance to the theatre. QTc (best monitored in lead II) ${ }^{2}$ was added with AAGBI monitoring. All the measures were taken to avoid hypothermia, because it can provoke QT prolongation. ${ }^{1,3}$

To attenuate the laryngoscopic response fentanyl was used and intubated after full relaxation. Due to the availability and familiarity, fentanyl was selected from esmolol and clonidine.

Propofol was selected as induction agent. Thiopentone, etomidate both can prolong QT and ketamine and midazolam can potentiate sympathetic properties and both have slow onset and risk of inadequate depth of anaesthesia. ${ }^{5}$ All the inhalational agents would prolong the QT interval in healthy. Isoflurane in beta blocked patients shortened the QT interval during maintenance. Therefore, isoflurane was used. ${ }^{3}$ Even though $\mathrm{N}_{2} \mathrm{O}$ is a sympathomimetic, it has been used safely in LQTS. ${ }^{4}$ Total intravenous anaesthesia would be the ideal in LQTS, unavailability of TCI pumps for paediatric patients were the limiting factor for its use in this situation.

Avoidance of sympathetic activation by adequate pain relief, adequate depth of anaesthesia and maintenance of normocarbia, normoxia and normoglycemia were concerned. ${ }^{3}$
From opioids sufentanil and methadone should be avoided (prolongs QT time). Adrenaline additives, cocaine and ketamine also should be avoided due to their sympathetic activity.

Depolarising neuromuscular agent (succinylcholine) and non-depolarising blocker pancuronium would prolong the QT interval. ${ }^{2}$ Except for the effects of histamine release, atracurium can be used. Vecuronium and rocuronium are also safe but cis-atracurium would be the ideal. Valsalva effects can precipitate QT prolongation, therefore high peak airway pressures, prolonged inspiratory time, end inspiratory pauses and extremely high end expiratory pressures were avoided. ${ }^{3}$ Since this patient is beta blocked, volume status needed to be optimised. ${ }^{3}$ Metaraminol and phenylephrine would be better option of sympathomimetic compared to ephedrine, adrenaline, noradrenaline and isoprenaline which prolong the QT time. ${ }^{2}$ Domperidone and ondansetron were avoided, if necessary, dexamethasone and metoclopramide can be safely used.

In the case of ' $\mathrm{Tdp}$ ', $\mathrm{MgSO}_{4} 30 \mathrm{mg} / \mathrm{kg}$ is given over $2 \mathrm{~min}$ and can be repeated. ${ }^{2}$ Other antiarrhythmics like amiodarone cannot be used since it prolongs QT interval. Pharmacokinetic and dynamic variations in drug combination may have a risk. Temporary transvenous pacing will be done if it is sustained.

\section{Recovery}

Acetylcholinesterase inhibitors and anticholinergics precipitate prolongation of QT by the effect of tachycardia and added with sympathetic stimulation at emergence. Sugammadex appears safe. Initial dose of atracurium would have metabolised by Hoffman degradation by the time of recovery and spontaneous breathing started, therefore reversal agents were avoided.

Since there is a risk of precipitating arrhythmias during immediate postoperative period, the patient was sent to the ICU for observation.

\section{Conclusion}

Adequate medical and electrophysiological optimisation with avoidance of all possible precipitating factors during perioperative period with vigilant monitoring would manage LQTS successfully. 
Fernando et al. Sri Lankan Journal of Anaesthesiology: 28(2):146-149 (2020)

\section{References}

1. O'hare M, Maldonado Y, Munro J, Ackerman MJ, Ramakrishna H, Sorajja D. Perioperative management of patients with congenital or acquired disorders of the QT interval. British journal of anaesthesia. 2018; 17:1-16 https://doi.org/10.1016/j.bja.2017.12.040 PMid:29576105

2. Levy D, Bigham C, Tomlinson D. Anaesthesia for patients with hereditary arrhythmias; part 2: congenital long QT syndrome and arrhythmogenic right ventricular cardiomyopathy. BJA Education. 2018;18(8)1-8: https://doi.org/10.1016/j.bjae.2018.04.005

3. Booker PD, Whyte SD, Ladusans EJ. Long QT syndrome and anaesthesia. British Journal of Anaesthesia. 2003;90(3):349-66.

https://doi.org/10.1093/bja/aeg061

PMid:12594150

4. Hondeghem LM, Carlsson L, Duker G. Instability and triangulation of the action potential predict serious proarrhythmia, but action potential duration prolongation is antiarrhythmic. Circulation. 2001;103(15):200413.

https://doi.org/10.1161/01.CIR.103.15.2004

PMid:11306531

5. Whyte SD, Booker PD, Buckley DG. The effects of propofol and sevoflurane on the QT interval and transmural dispersion of repolarization in children. Anesthesia \& Analgesia. 2005;100 (1):71-7.

https://doi.org/10.1213/01.ANE.0000140781.183 $\underline{91.41}$

PMid:15616054 